\title{
FERMI-LAT DETECTION OF PULSED GAMMA-RAYS ABOVE 50 GeV FROM THE VELA PULSAR
}

\author{
Gene C. K. Leung ${ }^{1}$, J. Takata ${ }^{1}$, C. W. NG ${ }^{1}$, A. K. H. Kong ${ }^{2}$, P. H. T. Tam ${ }^{2}$, C. Y. Hui ${ }^{3}$, and K. S. Cheng ${ }^{1}$ \\ ${ }^{1}$ Department of Physics, The University of Hong Kong, Pokfulam Road, Hong Kong; gene930@ connect.hku.hk, takata@hku.hk \\ 2 Institute of Astronomy and Department of Physics, National Tsing Hua University, Hsinchu, Taiwan \\ ${ }^{3}$ Department of Astronomy and Space Science, Chungnam National University, Daejeon, Korea \\ Received 2014 August 26; accepted 2014 October 19; published 2014 December 1
}

\begin{abstract}
The first Fermi-Large Area Telescope (LAT) catalog of sources above $10 \mathrm{GeV}$ reported evidence of pulsed emission above $25 \mathrm{GeV}$ from 12 pulsars, including the Vela pulsar, which showed evidence of pulsation at $>37 \mathrm{GeV}$ energy bands. Using 62 months of Fermi-LAT data, we analyzed the gamma-ray emission from the Vela pulsar and searched for pulsed emission above $50 \mathrm{GeV}$. Having confirmed the significance of the pulsation in $30-50 \mathrm{GeV}$ with the $H$ test ( $p$-value $\sim 10^{-77}$ ), we extracted its pulse profile using the Bayesian block algorithm and compared it with the distribution of the five observed photons above $50 \mathrm{GeV}$ using the likelihood ratio test. Pulsation was significantly detected for photons above $50 \mathrm{GeV}$ with a $p$-value of $=3 \times 10^{-5}(4.2 \sigma)$. The detection of pulsation is significant above $4 \sigma$ at $>79 \mathrm{GeV}$ and above $3 \sigma$ at $>90 \mathrm{GeV}$ energy bands, making this the highest energy pulsation significantly detected by the LAT. We explore the non-stationary outer gap scenario of the very high-energy emissions from the Vela pulsar.
\end{abstract}

Key words: gamma rays: stars - pulsars: individual (PSR J0835-4510) - radiation mechanisms: non-thermal

Online-only material: color figures

\section{INTRODUCTION}

The mechanism of the GeV gamma-ray emissions from pulsars remains to be solved since the discovery of gamma-ray emitting pulsars (Crab and Vela) in the 1970s. In the past several years, the observations of pulsar emissions above $10 \mathrm{GeV}$ by the Fermi-Large Area Telescope (Fermi-LAT) and groundbased Cherenkov telescopes have created a breakthrough in the understanding of the nature of $\mathrm{GeV}$ gamma-ray emissions from the Crab pulsar. In particular, the detection of the pulsed emission up to $400 \mathrm{GeV}$ by the MAGIC collaboration (Aliu et al. 2008; Aleksic et al. 2014) and the VERITAS Collaboration et al. (2011) were unexpected by the standard curvature radiation scenario of the outer magnetospheric gap. The observed spectrum between $100 \mathrm{MeV}$ and $400 \mathrm{GeV}$ is better described by a broken power law than the power law with exponential cutoff in the standard curvature radiation scenario. This result suggests that the inverse-Compton scattering process produces emissions above $10 \mathrm{GeV}$ in the Crab pulsar (Aleksic et al. 2011; Aharonian et al. 2012).

In the first Fermi-LAT catalog of sources above $10 \mathrm{GeV}$ (1FHL, Ackermann et al. 2013), 20 pulsars were found to show pulsed emissions ( $p$-value $\leqslant 0.05$ ) in the energy range $>10 \mathrm{GeV}$, including 12 pulsars showing pulsed emissions at $>25 \mathrm{GeV}$ ( $\mathrm{Saz}$ Parkinson et al. 2012). 1FHL also reported evidence of pulsation from the Vela pulsar at $>37 \mathrm{GeV}$ and two other pulsars (PSRs J0614-3329 and J1954+2836) at >60 GeV. Recently, pulsed emission of the Vela pulsar above $30 \mathrm{GeV}$ was also reported by the H.E.S.S. collaboration (2014), with a mean photon energy of $40 \mathrm{GeV}$.

Given the high significance detection of the Vela pulsar in 1FHL (e.g., $T S=72$ in 30-100 GeV), it is a good candidate for studying pulsed emission at high energy. The recent release of the reprocessed Pass 7 (P7REP) LAT data provides significant improvement over Pass 7 (P7) in the direction reconstruction of high-energy photons $>3 \mathrm{GeV}$ as well as improvement in energy reconstruction (Bregeon et al. 2013). For instance, the 95\% $(68 \%)$ containment angle for "Source" class events at $\sim 50 \mathrm{GeV}$ is reduced by $\sim 40 \%$ from $0.8(0.2)$ in $\mathrm{P} 7$ to 0.5 (0.12) in P7REP. The reduced point-spread function at high energy implies that the number of background photons, primarily from Vela-X in this case, inside the $95 \%$ containment angle of the target source is also reduced, leading to an enhanced signal-to-noise at high energy.

In this paper, we report on the analysis of 62 months of FermiLAT data and the high significance $(4.2 \sigma)$ detection of pulsed emissions at $>50 \mathrm{GeV}$ from the Vela pulsar. The detection of pulsation is significant above $4 \sigma$ at $>79 \mathrm{GeV}$ and above $3 \sigma$ at $>90 \mathrm{GeV}$ energy bands.

\section{OBSERVATIONS AND DATA ANALYSIS}

In this study, we analyzed Fermi-LAT data collected for 62 months, from 2008 August 4 to 2013 October 18. The data was reduced and analyzed using the Fermi Science Tools package (v9r32p5), available from the Fermi Science Support Center. ${ }^{4}$ We selected only events in the reprocessed Pass 7 "Source" class and used the P7REP_SOURCE_V15 IRFs. To reduce contamination from the Earth's albedo, we excluded time intervals when the zenith angle of the ROI was greater than $100^{\circ}$ or the LAT's rocking angle was greater than $52^{\circ}$. We calculated the pulse phases using the TEMPO2 plugin for Fermi, with the timing model of the Vela pulsar available from FermiLAT Multiwavelength Coordinating Group ${ }^{5}$ (Ray et al. 2011), which covers the entire span of the observation.

\subsection{Spectral Analysis}

We performed binned likelihood analyses using the gtlike tool in the region surrounding the Vela pulsar. We selected photons between 0.1 and $300 \mathrm{GeV}$ within a $20^{\circ} \times 20^{\circ} \mathrm{ROI}$ centered at the position of the Vela pulsar. We modeled the diffuse backgrounds with gll_iem_v05_rev1.fit and iso_source_v05.txt. We

\footnotetext{
4 http://fermi.gsfc.nasa.gov/ssc/data/analysis/software/

5 https://confluence.slac.stanford.edu/display/GLAMCOG/LAT+Gammaray+Pulsar+Timing+Models
} 


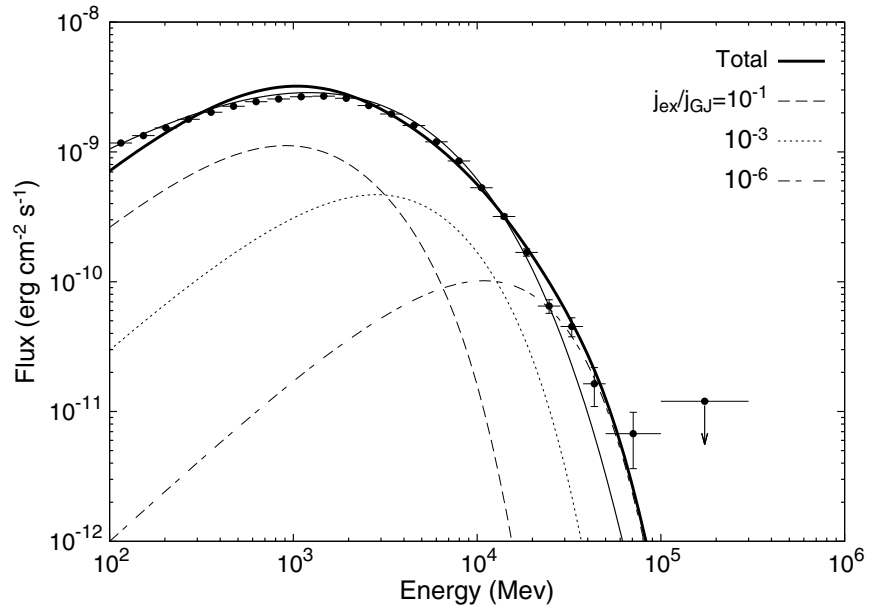

Figure 1. Phase-averaged gamma-ray spectrum of the Vela pulsar. The thick solid line shows the spectrum superposed on the emissions from the outer gap structures with the different injection currents. The result is for power index $p=0.6$ as the distribution of the external current (Equation (3)). The dashed, dotted, and dashed-dotted lines show individual contribution of the outer gap with $j_{\mathrm{ex}} / j_{\mathrm{GJ}}=10^{-1}, 10^{-3}$, and $10^{-6}$, respectively. We assume the inclination angle $\alpha=65^{\circ}$. The thin solid line shows the observational fit described by Equation (1).

included 2FGL sources (Nolan et al. 2012) within $15^{\circ}$ of the ROI center in the model. Only the spectral parameters of sources within $8^{\circ}$ of the ROI center were left free, while others were fixed to the catalog values. Since the publication of 2 FGL, extended emission from two nearby supernova renmants, Vela-Jr (Tanaka et al. 2011) and Puppis A (Hewitt et al. 2012), have been reported. The spatial and spectral models of the PWN Vela-X was also updated (Grondin et al. 2013). To model these extended sources, we first performed an off-pulse analysis for the region. We selected photons in the phase interval 0.8-1.0 (see Figure 3) for the off-pulse analysis as in Grondin et al. (2013).

We replaced the seven point sources in 2FGL that were possibly associated to Vela-Jr and Puppis A (Nolan et al. 2012) with the best-fit uniform circular disk spatial templates and simple power laws reported in Tanaka et al. (2011) and Hewitt et al. (2012). For Vela-X, we replaced the uniform circular disk model in 2FGL with the best-fit uniform elliptical disk template and broken power law reported by Grondin et al. (2013). Only the flux normalization parameters were left free. The off-pulse analysis was done with the Vela pulsar removed.

The off-pulse fit results, after correcting for exposure difference, were then passed to a phase-averaged fit. The fluxes of the three extended sources were fixed to the exposure-corrected off-pulse values. The Vela pulsar was modeled with a power law with exponential cutoff

$$
\frac{d N}{d E}=N_{0}\left(\frac{E}{E_{0}}\right)^{-\Gamma} \exp \left[-\left(\frac{E}{E_{\text {cut }}}\right)^{b}\right] .
$$

The best-fit values for the phase-averaged spectrum are $\Gamma=$ $1.086 \pm 0.004, E_{\text {cut }}=383 \pm 5 \mathrm{MeV}$, and $b=0.510 \pm 0.002$. The best fit function is overplotted in Figure 1.

Spectral points were obtained by performing likelihood fits in individual energy bands, fitting only the normalization parameters of the Vela pulsar and point sources within $4^{\circ}$, modeled as power laws, and the diffuse backgrounds. All other sources were fixed to the best-fit full band values. For the energy bands $50-100 \mathrm{GeV}$ and $100-300 \mathrm{GeV}$, the normalization parameter of Vela-X was also left free. The

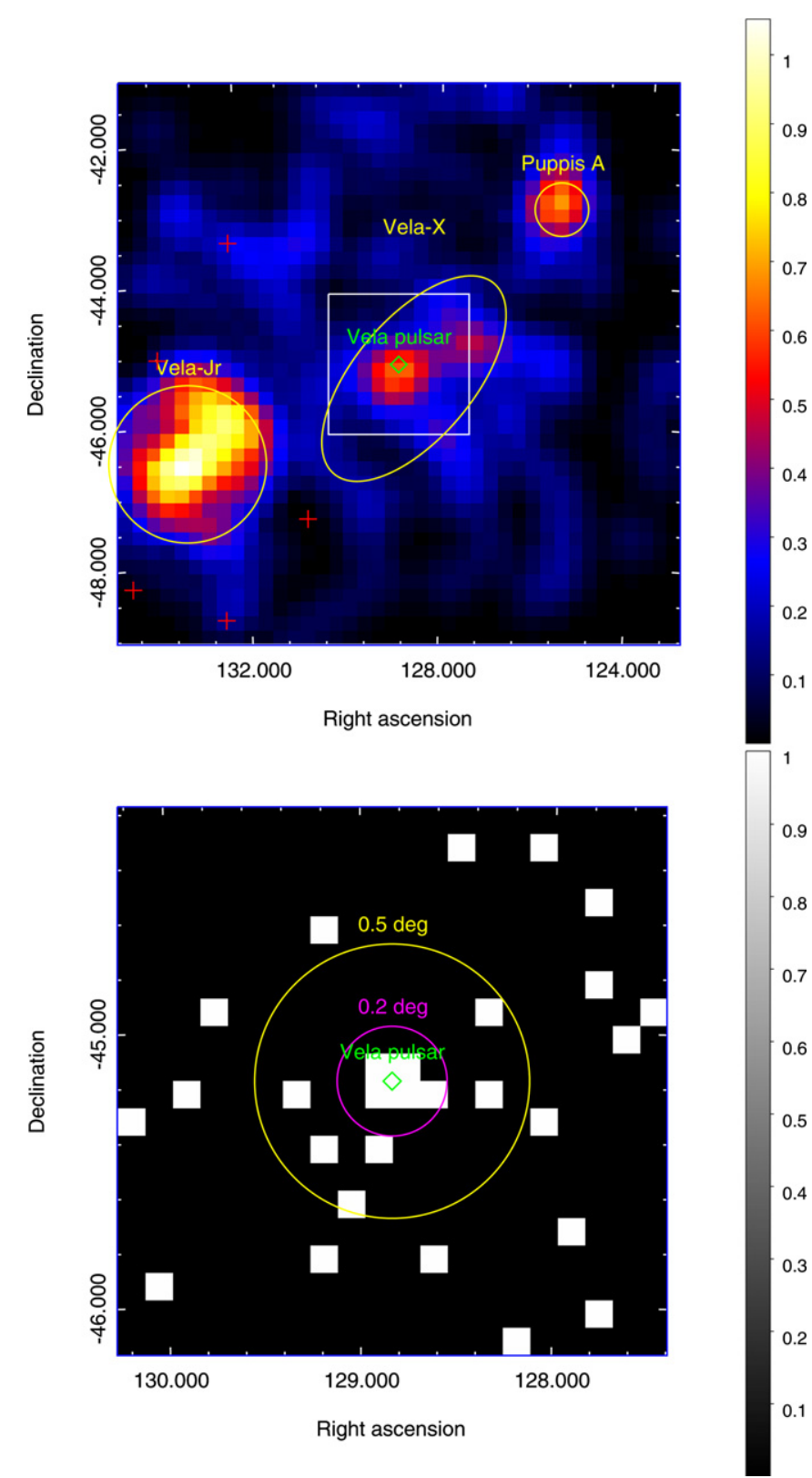

Figure 2. Upper panel: smoothed counts map of the $8^{\circ} \times 8^{\circ}$ region with $0.2 \times 0.2$ pixel size. The Vela pulsar is labeled with a green diamond, $2 \mathrm{FGL}$ sources with red crosses, and extended sources with yellow ellipses. The white square represents the region shown in the lower panel. Lower panel: counts map of the $2^{\circ} \times 2^{\circ}$ region with $0.1 \times 0$. 1 pixel size. No smoothing is applied. The five photons with $P_{\mathrm{PSR}}>\max \left(P_{\mathrm{PWN}}, P_{\mathrm{GAL}}\right)$ are all located within the 0.2 radius circle.

(A color version of this figure is available in the online journal.)

results are shown in Figure 1. The Vela pulsar is detected in $50-100 \mathrm{GeV}$ with a TS value of $9.5(3.1 \sigma)$ and a photon flux of $(3.17 \pm 1.78) \times 10^{-11}$ photons $\mathrm{cm}^{-2} \mathrm{~s}^{-1}$. In $100-300 \mathrm{GeV}$, the TS value of the Vela pulsar drops to $2.4(1.5 \sigma)$ and the $95 \%$ c.l. upper limit was shown.

A spectral fit was performed in $50-300 \mathrm{GeV}$. The source model was the same as above, except that the Vela pulsar and Vela-X were now modeled as power laws with the normalization factors and spectral indices left free. The Vela pulsar was detected with a TS value of $11.7(3 \sigma)$, with a photon index of $-2.53 \pm 0.98$ and a photon flux of $(4.27 \pm 2.13) \times$ $10^{-11} \mathrm{ph} \mathrm{cm}^{-2} \mathrm{~s}^{-1}$. Figure 2 (upper panel) shows the smoothed 


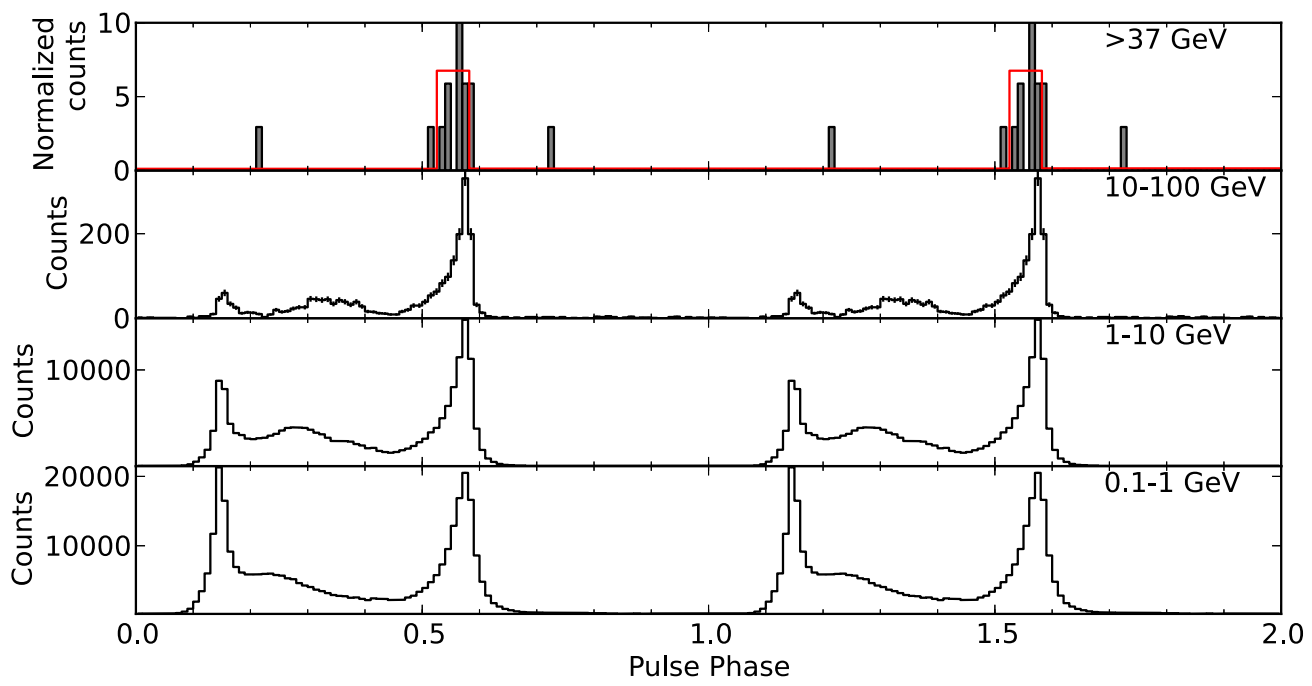

Figure 3. (a) Weighted light curve in 50-300 GeV. (b) Folded light curve in $30-50 \mathrm{GeV}$ with a 0.4 radius aperture. The black histogram represents the observed counts and the red line represents the Bayesian block decomposition. Folded light curves in (c) $10-100 \mathrm{GeV}$, (d) $1-10 \mathrm{GeV}$, and (e) $0.1-1 \mathrm{GeV}$ with a $1^{\circ}$ radius aperture.

(A color version of this figure is available in the online journal.)

counts map above $50 \mathrm{GeV}$ in a $8^{\circ} \times 8^{\circ}$ region. The emission around the position of the Vela pulsar is point-like and distinct from that of Vela-X. In contrast, the emission from Vela-Jr, with a spatial template of comparable size, is visibly extended.

\subsection{Pulsation Search}

\subsubsection{Weighted H Test}

Given the significant detection of the Vela pulsar in 50-300 GeV, we performed a pulsation search in this energy range. We applied a weighted $H$ test (Kerr et al. 2011) to this energy range. Each photon within $4^{\circ}$ from the Vela pulsar was weighted by its probability of having originated from the pulsar $\left(P_{\mathrm{PSR}}\right)$, calculated using the gtsrcprob tool. We used the spectral model obtained from the spectral fit in $50-300 \mathrm{GeV}$ described in Section 2.1 to calculate the probabilities. The sum of probabilities $\sum P_{\mathrm{PSR}}=6.3$ and the weighted $\mathrm{H}$ statistic is 15.4 , corresponding to a $p$-value of 0.002 or $3.1 \sigma$. Figure $3(\mathrm{a})$ shows the weighted light curve in $50-300 \mathrm{GeV}$. The weighted $H$ test for higher energy ranges returned $H<15$, i.e., below $3 \sigma$.

As noted in 1FHL, the $H$ test (de Jager et al. 1989; Kerr et al. 2011) is not the most sensitive tool for the pulsation search on known pulsars at high energy, as it does not utilize any prior knowledge of the pulse shape. Therefore, we apply a likelihood ratio test in our search. The test compares the distribution in pulse phase of high-energy photons with a known pulse profile in lower energies, and determines whether the high-energy photons are better described by the known pulse profile or a uniform distribution. For this test to be most effective, it is necessary that the lower-energy pulse profile should satisfactorily reflect the expected distribution of high-energy photons.

\subsubsection{Selection of Low-energy Pulse Profile}

As shown in previous studies (e.g., Abdo et al. 2010, 2013), the pulse profile of the Vela pulsar is strongly dependent on energy. Figure 3(c) to (e) shows the folded light curves of the Vela pulsar in three energy bands: $0.1-1 \mathrm{GeV}, 1-10 \mathrm{GeV}$, and $10-100 \mathrm{GeV}$. With increasing energy, $\mathrm{P} 1$ weakens significantly, with the peak heights ratio $\mathrm{P} 1: \mathrm{P} 2$ decreasing by a factor of $\sim 5$ from $0.1-1 \mathrm{GeV}$ to $10-100 \mathrm{GeV}$. The bridge structure also shifts toward P2 and reduces in height. Therefore, it is expected that the photons above $50 \mathrm{GeV}$ should follow a distribution more similar to that in 10-100 GeV and concentrate around P2, and it is not appropriate to apply the full energy pulse profile in the likelihood ratio test.

In order to obtain a pulse profile that better reflects the highenergy behavior, we need to use an energy range high enough to reflect the expected distribution of the $>50 \mathrm{GeV}$ photons, but low enough to demonstrate a statistically significant pulse profile. We extracted the folded light curve in $30-50 \mathrm{GeV}$ and performed the unweighted $H$ test (de Jager et al. 1989). We experimented with different aperture radii in steps of 0.1 and found that a 0.4 radius maximizes the $H$ statistic, giving $H=440$ ( $p$-value $\sim 10^{-77}$ ). This represents a statistically significant pulse profile to be used in the likelihood ratio test.

We then employed the Bayesian block algorithm (Scargle et al. 2013) to identify statistically significant variation features in the folded light curve in $30-50 \mathrm{GeV}$ in a 0.4 radius aperture, using a false positive threshold of $0.5 \%$. Since the Bayesian block algorithm assumes Poisson statistics, it can only be applied to unweighted photons. The folded light curve in $30-50 \mathrm{GeV}$ is represented by a block spanning the phase interval from 0.5159 to 0.5870 . Figure 3 (b) shows the $30-50 \mathrm{GeV}$ folded light curve overlaid with the Bayesian blocks. This block function was taken to be the low-energy pulse profile in the likelihood ratio test.

\subsubsection{Photon Selection Above $50 \mathrm{GeV}$}

To select $>50 \mathrm{GeV}$ photons for the likelihood ratio test, we also calculated the probabilities of coming from Vela-X $\left(P_{\mathrm{PWN}}\right)$ and the Galactic diffuse emission $\left(P_{\mathrm{GAL}}\right)$ for photons within $4^{\circ}$ from the Vela pulsar, following the procedures described in Section 2.2.1. Since the Vela pulsar is completely embedded in Vela-X as seen by the LAT, the major sources of background contamination are Vela-X and the Galactic diffuse emission. Therefore, we selected only photons with $P_{\mathrm{PSR}}>\max \left(P_{\mathrm{PWN}}, P_{\mathrm{GAL}}\right)$.

A total of five photons have $P_{\mathrm{PSR}}>\max \left(P_{\mathrm{PWN}}, P_{\mathrm{GAL}}\right)$. Figure 2 (lower panel) displays the counts map above $50 \mathrm{GeV}$. All the five photons are located within the 0.2 circle centered at the Vela pulsar. We also note that all five photons fall into the highest quality "Ultraclean" class of events, meaning that they 


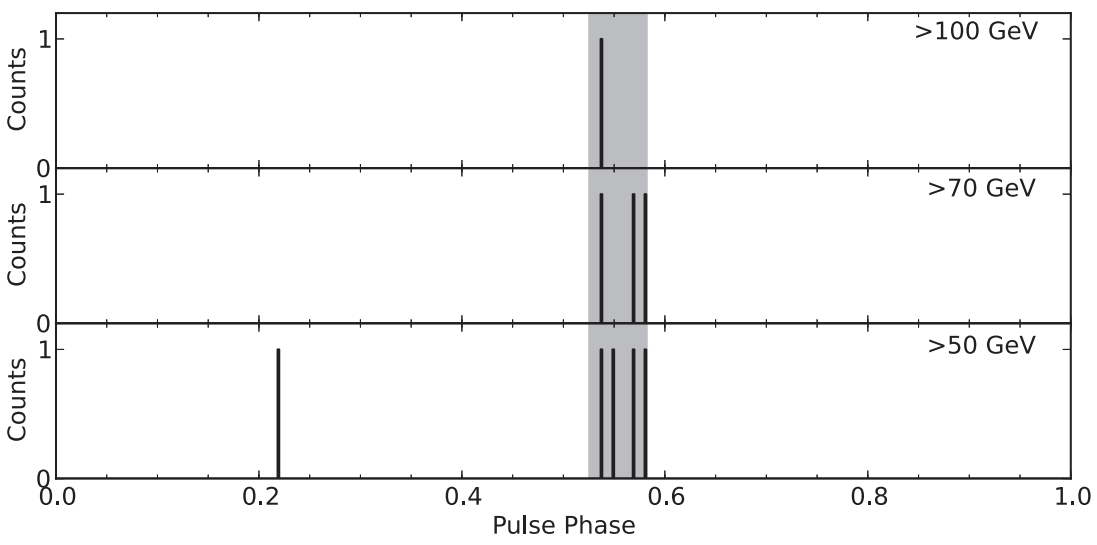

Figure 4. Folded light curves for 50-300 GeV (bottom), 70-300 GeV (middle), and 100-300 GeV (top). The shaded area represents the on-peak interval decided by Bayesian blocks at $30-50 \mathrm{GeV}$.

Table 1

Energy, Arrival Time, Angular Separation from the Vela Pulsar, Pulse Phase, and Source Probability of $>50 \mathrm{GeV}$ Photons with $P_{\mathrm{PSR}}>\max \left(P_{\mathrm{PWN}}, P_{\mathrm{GAL}}\right)$ in Ascending Order of Energy

\begin{tabular}{lccccccc}
\hline \hline $\begin{array}{l}E_{\gamma} \\
(\mathrm{GeV})\end{array}$ & $\begin{array}{c}\text { Time } \\
(\mathrm{MJD})\end{array}$ & $\begin{array}{c}\Delta \theta \\
\left({ }^{\circ}\right)\end{array}$ & Pulse Phase & $P_{\mathrm{PSR}}$ & $D_{E \geqslant E_{\gamma}}$ & $p_{E \geqslant E_{\gamma}}$ & $\sigma_{E \geqslant E_{\gamma}}$ \\
\hline 51.3 & 55050.8 & 0.074 & 0.548 & 0.940 & 16.3 & $3.3 \times 10^{-5}$ & $4.2 \sigma$ \\
55.9 & 56149.3 & 0.136 & 0.219 & 0.707 & 11.5 & $4.2 \times 10^{-4}$ & $3.5 \sigma$ \\
79.5 & 56317.2 & 0.034 & 0.581 & 0.986 & 15.9 & $6.0 \times 10^{-5}$ & $4.0 \sigma$ \\
91.0 & 56437.5 & 0.011 & 0.569 & 0.994 & 10.6 & $1.4 \times 10^{-3}$ & $3.3 \sigma$ \\
208.5 & 55154.1 & 0.092 & 0.537 & 0.922 & 5.3 & $2.8 \times 10^{-2}$ & $2.2 \sigma$ \\
\hline
\end{tabular}

Notes. Three glitches occurred at MJD 53959.9, MJD 55408.8, and MJD 56555.8. The $D$ value, $p$-value, and significance of pulsation at the energy range bounded below by $E_{\gamma}$ of each photon are also shown.

have the strictest rejection against cosmic ray events, which are more common at higher energies. Figure 4 shows the folded light curves in three energy bands: 50-300 GeV, 70-300 GeV, and 100-300 GeV. The shaded area represents the phase interval spanned by the Bayesian block in 30-50 GeV. Four of the five photons are located within the Bayesian block and are distributed across all three energy bands. The highest energy photon within the Bayesian block is at $209 \mathrm{GeV}$. Table 1 lists the energy, arrival time, angular separation from the Vela pulsar, pulse phase, and source probability of the five photons. We note that one glitch occurred at MJD 53959.9 (Yu et al. 2013) before the start of the observation, and two other glitches were recorded during the span of the observation at MJD 55408.8 and MJD 56555.8 in the timing model. None of the selected photons was detected near a glitch epoch.

\subsubsection{Likelihood Ratio Test}

We performed a maximum likelihood fit to the $>50 \mathrm{GeV}$ folded light curve using a probability distribution of a block function

$$
\operatorname{PDF}(\phi)= \begin{cases}1-s+\frac{s}{\phi_{1}-\phi_{0}} & \text { if } \phi_{0} \leqslant \phi \leqslant \phi_{1} \\ 1-s & \text { otherwise, }\end{cases}
$$

where $\phi$ is the pulse phase, $\phi_{0}$ and $\phi_{1}$ are fixed to the edges of the Bayesian block in 30-50 GeV, and $s$ is a free parameter in $[0,1]$. We also performed a fit with the null hypothesis of a uniform distribution, i.e., $s=0$. Using the likelihood ratio test, we obtained the test statistic, $D=-2 \Delta \log ($ Likelihood $)=16.3$ for $E>50 \mathrm{GeV}$. We scanned up in energy by removing the lowest-energy photon each time and calculating the $D$ value. The $D$ values are listed in Table 1 .
Since the Wilk's theorem, which translates $D$ to $p$-values, assumes infinite statistics, we calculated the $p$-values with Monte Carlo simulations. In each Monte Carlo realization, $N$ phases, where $N$ is drawn from a Poisson distribution with the mean equal to the observed number of photons, were randomly generated in $[0,1]$. Then the same analysis was performed to the simulated data to calculate the $D$ value. The $p$-values from Monte Carlo simulation agree with Wilk's theorem and are listed in Table 1. The significance of pulsation is above $4 \sigma$ for $>51$ and $>79 \mathrm{GeV}$, and above $3 \sigma$ at $>90 \mathrm{GeV}$ energy bands. We note that the pulsation significance for $>55 \mathrm{GeV}$ is below $4 \sigma$, due to the contribution of an off-pulse photon at $55.9 \mathrm{GeV}$. We also note that, however, this particular photon has a smaller probability $(\sim 0.7)$ to have originated from the Vela pulsar than the other four photons $(>0.9)$.

\section{DISCUSSION}

As shown in Figure 1, the observed flux above $10 \mathrm{GeV}$ of the Vela pulsar decreases slower than a simple exponential function. Previous emission models that invoke the curvature radiation process for the $\mathrm{GeV}$ emissions have in general predicted a flux at the 50-100 GeV smaller than the observed flux level of $10^{-12} \mathrm{erg} \mathrm{cm}^{-2} \mathrm{~s}^{-1}$. Abdo et al. (2010) proposed that a subexponential cutoff in the observed spectrum can be understood as the superposition of several power low plus exponential cutoff functions with varying the photon index and the cutoff energy.

In the section, we argue the model that the outer gap accelerator is switching between a number of states, and that superposition of the emissions from the different states of the outer gap make the observed spectrum of Fermi. There is a wide range of variability times scale in the radio emissions from the pulsar (e.g., Kramer et al. 2002; Lyne et al. 2010; Keane 2013). The 
micro-second variations seen in single pulse could be produced by spatial fluctuation in the emission region. The pulse-to-pulse variations on the timescale of milliseconds to seconds likely represent the timescale of the temporal variation of the structure of the emission region. The longer timescale (seconds to years) variations associated with the model switching and nulling, which sometimes accompany the variations of the spin down rate, could be related with the changes of entire magnetosphere. The higher-energy observations also found the mode switches in the X-ray emission properties of PSR B0943+10 (Hermsen et al. 2013) and in the GeV gamma-ray emission properties of PSR J2021+4026 (Allafort et al. 2013). These multi-wavelength observations suggest that the switching between a number of state of magnetosphere is probably a generic feature of the pulsars.

The active outer gap should require the external currents $\left(j_{\text {ex }}\right)$ that are injected into the outer gap at the boundaries because they initiate the gamma-ray emissions and subsequent paircreation cascade process. In our model, the outer gap structure and the properties of the curvature radiation depend on how large currents are injected into the gap. For example, the outer gap size can develop until the pair-creation process creates the gap current of the order of the Goldreich-Julian value $j_{\mathrm{GJ}} \sim \Omega B / 2 \pi$ (Takata et al. 2004; Hirotani 2006). The outer gap with a smaller external current has a larger gap size and a larger electric field, and produces harder gamma-rays. If the external current, which may be originated from the polar cap region, is temporally variable, the observed gamma-ray spectrum will be the superposition of the emissions from various gap states.

We consider that the outer gap (Cheng et al. 1986; Takata et al. 2006) produces the $\mathrm{GeV}$ emissions; in the model the charge depletion from the Goldreich-Julian charge density causes the electric field along the magnetic field line, which accelerates the electrons and positrons to Lorentz factor of $\sim 10^{7}$. We assume the dipole magnetic field in the magnetosphere and solve three-dimensional (3D) structure of the gap (J. Takata et al. in preparation); (1) the particle acceleration process by the electric field, (2) the curvature radiation process, and (3) the paircreation process between the gamma-rays and thermal X-rays from the neutron star surface. Our 3D model is the expansion of a two-dimensional study developed by Takata et al. (2006, 2008) and Takata \& Chang (2009), which discussed the phaseaveraged spectra of the pulsars measured by the EGRET.

In our model, we assume that the observed gamma-ray spectrum is a superposition of the emissions from various stationary gap structures with various external currents. The model assumes that the timescale of the variability of the external current is of the order of or longer than the crossing timescale of the light cylinder radius $\left(r_{l c}\right), \tau \sim r_{l c} / c=p / 2 \pi$, with which the stationary outer gap structure for an external current is archived. Figure 1 compares the calculated spectra with the observations. In the calcualtion, we assumed the powerlaw distribution of the external current as

$$
I=K j_{\mathrm{ex}}^{p}, j_{\min }<j_{\mathrm{ex}}<j_{\max },
$$

where $K$ is the normalization factor and is determined from $\int\left(d I / d j_{\mathrm{ex}}\right) d j_{\mathrm{ex}}=1$, and we used $j_{\min }=10^{-6} j_{\mathrm{GJ}}$ and $j_{\max }=$ $0.2 j_{\mathrm{GJ}}$. In addition, we chose the power index $p \sim 0.6$, which reasonably reproduces the observed spectral cutoff behavior. In Figure 1, the calculated spectrum for an external current cannot explain the observed spectral shape in $100 \mathrm{MeV}-50 \mathrm{GeV}$, while the gamma-ray spectrum superposed by the different gap states better explains the observed cutoff behavior. We also find that the emissions above $20 \mathrm{GeV}$ can be explained by the outer gap with a very small injection current. In a subsequent paper, we will study dependency of the viewing geometry (inclination angle and viewing angle) on the predicted spectrum and will discuss the goodness of the model fitting for high-energy pulsars.

In summary, we detected pulsed gamma-ray emissions from the Vela pulsar at above $4 \sigma$ at $>79 \mathrm{GeV}$ and above $3 \sigma$ at $>90 \mathrm{GeV}$ energy bands using Fermi-LAT. This is difficult to explain using the previous stationary model in the pulsar magnetosphere. We proposed the model that the outer gap structure switches between a number of state. Future searches for pulsed emission above $50 \mathrm{GeV}$ in other pulsars will help us to understand the nature of the high-energy emission in the pulsar magnetosphere.

We thank anonymous referee, P. M. Saz Parkinson, and J. E. McEnery for useful comments and suggestions, and E. M. H. Wu, and J. H. K. Wu for providing the weighted H-test computing code. This work is partially supported by a 2014 GRF grant under HKU 17300814P and Seed Funds under HKU 20127159004 and 201310159026 . A.K.H.K. and P.H.T. are supported by the National Science Council of the Republic of China (Taiwan) through grants 100-2628-M-007-002-MY3 and 1002923-M-007-001-MY3, and 101-2112-M-007-022-MY3, respectively. C.Y.H. is supported by a research fund from Chungnam National University in 2014. J.T. acknowledges TIARA, operated under the ASIAA of Taiwan to use their PC cluster.

\section{REFERENCES}

Abdo, A. A., Ackermann, M., Ajello, M., et al. 2010, ApJ, 713, 154 Abdo, A. A., Ajello, M., Allafort, A., et al. 2013, ApJS, 208, 17 Ackermann, M., Ajello, M., Allafort, A., et al. 2013, ApJS, 209, 34 Aharonian, F. A., Bogovalov, S. V., \& Khangulyan, D. 2012, Natur, 482, 507 Aleksic, J., Alvarez, E. A., Antonelli, L. A., et al. 2011, ApJ, 742, 43 Aleksic, J., Ansoldi, S., Antonelli, L. A., et al. 2014, A\&A, 565, L12 Allafort, A., Baldini, L., Ballet, J., et al. 2013, ApJL, 777, 2

Aliu, E., Anderhub, H., Antonelli, L. A., et al. 2008, Sci, 322, 1221

Bregeon, J., Charles, E., \& Wood, M. 2013, arXiv:1304.5456

Cheng, K. S., Ho, C., \& Ruderman, M. 1986, ApJ, 300, 500

de Jager, O. C., Raubenheimer, B. C., \& Swanepoel, J. W. H. 1989, A\&A, 221,180

Grondin, M.-H., Romani, R. W., Lemoine-Goumard, M., et al. 2013, ApJ, 744,110

Hermsen, W., Hessels, J. W. T., Kuiper, L., et al. 2013, Sci, 339, 436

H.E.S.S. Collaboration. 2014, in Astroparticle Physics 2014: A Joint TeVPA/ iDM Conference, https://www.mpi-hd.mpg.de/hfm/HESS/201406-TeVPAAmsterdam-nofilm.pdf

Hewitt, J. W., Grondin, M.-H., Lemoine-Goumard, M., et al. 2012, ApJ, 759,89

Hirotani, K. 2006, ApJ, 652, 1475

Keane, E. F. 2013, in IAU Symp. 291, Neutron Stars and Pulsars: Challenges and Opportunities after 80 Years, ed. J. van Leeuwen (Cambridge: Cambridge Univ. Press), 295

Kerr, M. 2011, ApJ, 732, 38

Kramer, M., Johnston, S., \& Van Straten, W. 2002, MNRAS, 334, 523

Lyne, A., Hobbs, G., Kramer, M., Stairs, I., \& Stappers, B. 2010, Sci, 329, 408 Nolan, P. L., Abdo, A. A., Ackermann, M., et al. 2012, ApJS, 199, 31

Ray, P. S., Kerr, M., Parent, D., et al. 2011, ApJS, 194, 17

Saz Parkinson, P. M., et al. 2012, in Proc. AIP Conf. 1505, High Energy GammaRay Astronomy, ed. F. A. Aharonian, W. Hofmann, \& F. M. Rieger (Melville, NY: AIP), 293

Scargle, J. D., Norris, J. P., Jackson, B., \& Chiang, J. 2013, ApJ, 764, 167

Takata, J., \& Chang, H.-K. 2009, MNRAS, 392, 400

Takata, J., Chang, H.-K., \& Shibata, S. 2008, MNRAS, 386, 748

Takata, J., Shibata, S., \& Hirotani, K. 2004, MNRAS, 354, 1120

Takata, J., Shibata, S., Hirotani, K., \& Chang, H.-K. 2006, MNRAS, 366, 1310

Tanaka, T., Allafort, A., Ballet, J., et al. 2011, ApJL, 740, L51

VERITAS Collaboration., et al. 2011, Sci, 334, 69

Yu, M., Manchester, R. N., Hobbs, G., et al. 2013, MNRAS, 429, 688 